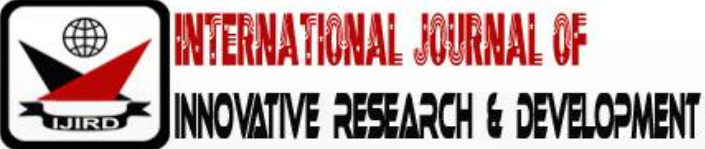

ISSN 2278 - 0211 (Online)

\section{Soft Computing: Optimization of Hybridized Neural Network Variant with Genetic Algorithm for Anomaly Detection}

\begin{tabular}{|c|} 
Ismaila Wasiu Oladimeji \\
Associate Professor, Department of Computer Science and Engineering, \\
Ladoke Akintola University of Technology, Ogbomoso, Nigeria \\
Ayodele Adefemi Lawrence \\
Student, Department of Computer Science and Engineering, \\
Ladoke Akintola University of Technology, Ogbomoso, Nigeria \\
Omidiora E. Olusayo \\
Professor, Department of Computer Science and Engineering, \\
Ladoke Akintola University of Technology, Ogbomoso, Nigeria \\
Falohun Adeleye S. \\
Associate Professor, Department of Computer Science and Engineering, \\
Ladoke Akintola University of Technology, Ogbomoso, Nigeria
\end{tabular}

\begin{abstract}
:
Objective: to experiment the performance of optimization of hybridized counter propagation neural network (CPNN) with genetic algorithm (GA) to detect anomaly in credit cards online transactions. Methods: One thousand three hundred transactional data from thirteen cardholders were simulated which contained a mix of genuine and fraudulent transactions. The system work flow includes data preparation, CPNN parameters optimization process, data classification and evaluation. Results: The performance of GA, CPNN, and CPNN-GA were evaluated based on prediction accuracy $\left(\mathrm{p}_{\mathrm{acc}}\right)$, false acceptance rate $\left(\mathrm{fa}_{\text {rate }}\right)$, false rejection rate $\left(\mathrm{fr}_{\text {rate }}\right)$, and equal error rate (eqrate). The results generated by GA anomaly detection system gave averages of $95.0 \%$, $0.0 \%, 10.0 \%$, and $5.0 \%$; CPNN anomaly detection system produced averages of $94.5 \%, 0.0 \%, 7.3 \%$ and $3.8 \%$ while CPNN-GA anomaly detection system gave $97.3 \%, 0.0 \%, 3.7 \%$ and $2.0 \%$ for $\mathrm{p}_{\text {acc, }} \mathrm{fa}_{\text {rate, }} \mathrm{fr}_{\text {rate }}$ and $\mathrm{eq}_{\text {rate }}$ respectively. The false alarm rate for the GA, CPNN and CPNN-GA are $0.83 \%, 1.35 \%$ and $0.61 \%$ respectively. Conclusion: A hybrid approach based on CPNN and GA provided results for enhanced accuracy in online transactions fraud detection system with lowest false alarm rate. The developed fraud detection system is critically important for security and economic operation in banking system.
\end{abstract}

Keywords: E-commerce, credit cards, CPNN, Genetic Algorithm, anomaly detection

\section{Introduction}

Outlier/anomaly detection refers to the problem of finding patterns in data that do not conform to expected behaviour. These nonconforming patterns are often referred to as anomalies, outliers, exceptions, aberrations, or peculiarities in different application domains. Of these, anomalies and outliers are two terms used most commonly in the context of anomaly detection, sometimes interchangeably. Anomaly detection finds extensive use in a wide variety of applications such as fraud detection for credit cards applications, intrusion detection for cyber-security, and military surveillance for enemy activities (Varun etal, 2009). Frauds can be broadly classified into three categories, i.e., traditional card related frauds, merchant related frauds and Internet frauds. The different types of methods for committing

credit card frauds can be found in (Patidar and Sharma, 2011), (Neda, etal, 2012).

The growing in the scale of e-commerce has become eye catching to criminals, and the numbers of fraudulent ecommerce transactions is escalating rapidly. Therefore, there has been an increase in the amount of attention given to the security of the payment systems used to process online transactions. Probably the main current concern of most Internet users relates to the confidentiality of payment card information, since there is a growing realisation that stolen card details can be used to make fraudulent transactions (Chan, etal, 1999). This work evaluated the performance of optimization of hybridized counter propagation neural networks (CPNN) with genetic algorithm (GA) in credit card anomaly detection. 


\section{Related Works}

Many researchers used different meta-learning algorithms to create hybrid credit card fraud detection system. Peter et al used an evolution-fuzzy detection system to classify suspicious and non-suspicious credit card transactions. This system composed of genetic programming (GP) search algorithm and a fuzzy expert system. It first clustered data into three groups, namely 'high', 'medium' and 'low'. GP part evolves a series of variable-length rules, which characterize the differences of data. They concluded that the use of evolution with fuzzy logic has higher accuracy. Devaki, etal (2014) proposed a credit card fraud detection using time series analysis. They used the periodic pattern in the spending behaviour of a cardholder to detect the anomalies in the transaction with respect to the analyses of the past history of transactions belonging to an individual cardholder. Firstly, distance-based method was used to detect whether the new incoming transaction is fraud or not and secondly, the next transaction is predicted by means of label prediction methodology and compared with the actual transaction, if there is deviation then it is detected to be a fraudulent transaction. If the particular transaction was considered as a fraud then the cardholder was asked to continue the transaction by asking a secret question, if the cardholder did not give correct answer then the transaction would not be declined. The approach decreased the false positive situation and hence it ensured that genuine transaction is not rejected. Panigrahi et al proposed a fusion system consisted of four components: rule-based filter, Dempster-Shafer adder, transaction history database and Bayesian learner. Rule-based filters first generates a suspicious score. If it could not determine whether an incoming transaction was fraud or not, Bayesian model further detected the transaction, and give the final result.

Pooja etal (2015), in their work made an attempt to detect fraudulent credit card transactions by using k-means along with genetic algorithm (an optimization technique). The k-means algorithm grouped the credit card transactions based on the independent attribute values. But, with the increase in input size, it resulted in anomalies. Hence genetic algorithm provided optimized detection of frauds which provided significant results. Oladele, et al (2018)did research work that produced Hybridized-Cross-Semi-Global Alignment to solve the problem of masquerade intrusion into data or information kept on the computer system by reducing the false positive alarm and false negative alarm which increases the true positive proportionately. The results produced hit rate of $90 \%$ and a $4.1 \%$ of false positive. Akhilomen in 2013 presented a model by using hybrid feature selection and anomaly detection algorithm in order to detect fraud in credit cards. In the study, the people who performed fraudulent activities in the field of credit cards were classified into; the buyers of credit cards information; Black hat hackers and the Thieve of credit cards. Since the use of credit card by card holders followed a fixed pattern, this fixed pattern could be extracted from a usual legal activity of card holders in 1 or 2 years. Thus, this pattern was compared to the use of process of card holder and in case of non-similarity in the pattern, the activity was considered illegal. Related work can be found in You et al (2016), Pouramirarsalani,et al (2017).

\subsection{Materials}

This section discussed the algorithms employed for developing anomaly detection.

\subsubsection{Counter-Propagation Networks}

The counter-propagation network combined a portion of the Kohonen self-organizing map (Kohonen, 1995) and Grossberg outstar structure (Grossberg, 1982). During learning, pairs of the input vector $\mathbf{X}$ and output vector $\mathbf{Y}$ were presented to the input and interpolation layers, respectively. These vectors propagate through the network in a counter flow manner to yield the competition weight vectors and interpolation weight vectors. Once these weight vectors become stable, the learning process is completed. The output vector $\mathbf{Y}^{\prime}$ of the network corresponding to the input vector $\mathbf{X}$ is then computed. The vector $\mathbf{Y}^{\prime}$ is intended to be an approximation of the output vector $\mathbf{Y}^{\prime}$ i.e.

$Y \approx Y-f(X)$.. (1)

The equations of the network are described briefly as follows.

$U_{j}=\left[U_{j i}\right]$

Equation 2 is the arbitrary initial competition weight vector for the $\mathrm{j}$-th neuron in the competition layer where $\mathrm{u}_{\mathrm{j} i \mathrm{is}}$ the weight connecting the $\mathrm{j}$-th neuron in the competition layer to the $\mathrm{i}$-th neuron in the input layer. The Euclidean distance between the input vector $\mathbf{X}$ and the competition weight vector $\mathbf{U} \mathbf{j}$ of the j-th neuron is calculated, i.e.:

$$
d_{j}=\left\|X-U_{j}\right\| \sqrt{\sum_{t=1}^{m}\left(x_{t}-u_{j t}\right)^{2}}
$$

Once the distance for each neuron has been calculated, the neuron with the shortest Euclidean distance to $\mathbf{X}$ is selected to represent the winning neuron. As a result of the competition, the output of the winning neuron is set to unity and the outputs of the other neurons are set to zero. Thus, the output of the j-th neuron in the competition layer can be expressed as:

$$
z_{j}=\left\{\begin{array}{cc}
1.0 & \text { if } d_{j}<d_{t} \text { for all } i \\
0.0 & \text { atherwise }
\end{array}\right.
$$

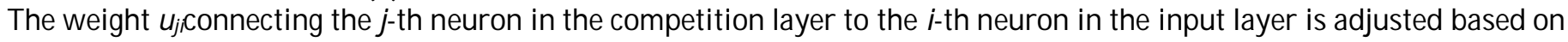
the Kohonen learning rule, i.e.:

$$
u_{i j}(p+1)=u_{j i}+\beta\left(x_{i}-u_{j i}(p)\right) z_{i} . .(5)
$$


Where $\beta$ is the learning coefficient and $p$ is the iteration number. After the competition weight vector $\mathbf{U}_{\mathbf{j}}$ stabilizes, the interpolation layer starts to learn the desired output vector $\mathbf{Y}$ by adjusting the interpolation weight vector. Let

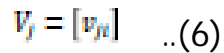

Equation 6 is the arbitrary initial interpolation weight vector for the $\mathrm{j}$-th neuron in the interpolation layer where $\mathrm{v}_{\mathrm{j} i \mathrm{is}}$ the weight connecting the $j$-th neuron in the interpolation layer to the $\mathrm{i}$-th neuron in the competition layer. The weight $\mathrm{v}_{\mathrm{ji}}$ is adjusted based on the Grossberg learning rule, i.e.

$$
v_{i j}(p+1)=v_{s i}+r\left(y_{t}-v_{n}(p)\right) s_{i} . .(7)
$$

Where $\gamma_{\mathrm{i}}$ is the learning coefficient. This is repeated until the interpolation weight vector $\mathbf{V}_{\mathrm{j}}$ converges to a preset value. The output vector $\mathbf{Y}^{\prime}$ of the network corresponding to the input vector $\mathbf{X}$ can be calculated using a weighted summation function. The j-th component $y_{j}^{\prime}$ of the output vector $\mathbf{Y}^{\prime}$ can be expressed as:

$y_{i}=\sum v_{p_{1}} z_{t}$

The counter-propagation network functions as a look-up table. The learning process associates the input vector with the corresponding output vector based on two well-known algorithms: the Kohonen self-organizing map for finding the most similar training vector and the Grossberg outstar map for projecting the corresponding output vector. Once the network is trained, the application of an input vector can quickly reduce the corresponding output vector.

\subsubsection{Genetic Algorithm (GA)}

A GA was created by specifying either an individual or a population of individuals for initialization. If a single individual is specified, the simple genetic algorithm cloned the individual that is specified to make its own population. In each generation the algorithm created an entirely new population of individuals by selecting from the previous population and then mating to produce the new offspring for the new population. This process continued until the stopping criteria were met.

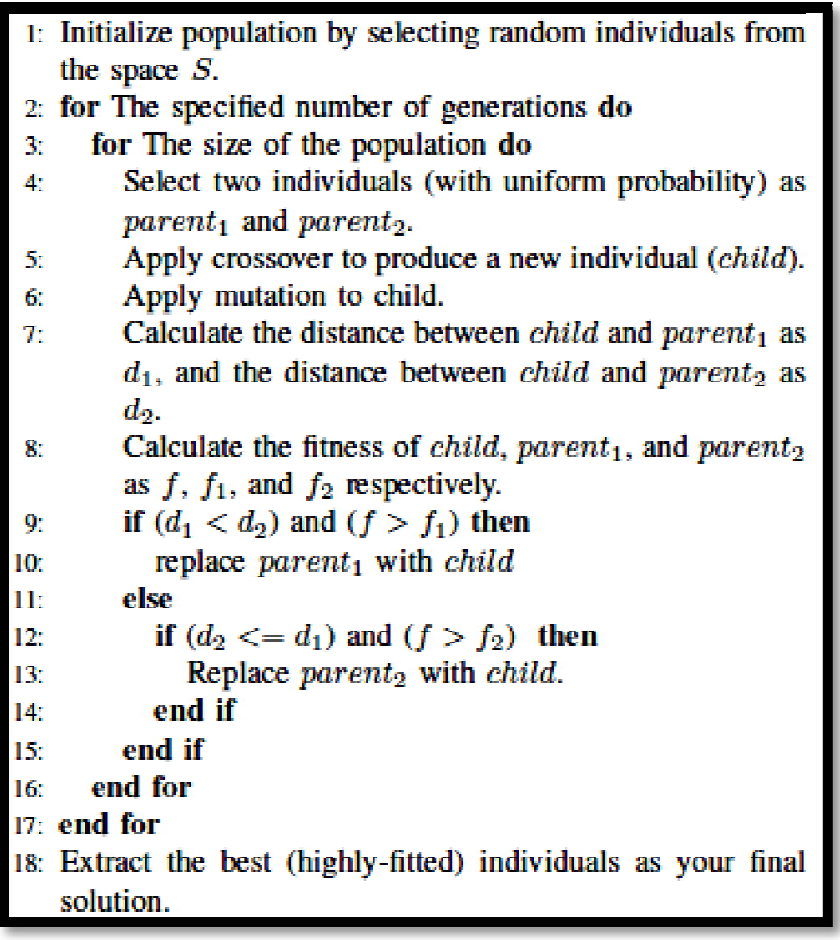

Figure 2: GA pseudocode Arifin S. (2011)

In order to set a time limit to the potential model runs, this study used the number of generations as the termination criterion. Probabilities of mutation and crossover were varied in this work, likewise the operators for selection. There was provision to change the operators for mutation and crossover. The GA operators are explained in the table 1; 


\begin{tabular}{|c|c|}
\hline GA operators & Description \\
\hline individual & $\begin{array}{l}\text { A single solution which can } \\
\text { be either chromosome/ } \\
\text { genotype and/ or phenotype }\end{array}$ \\
\hline Chromosome & $\begin{array}{l}\text { is the raw genetic } \\
\text { information that is dealt by } \\
\text { genetic algorithm. }\end{array}$ \\
\hline Populations & $\begin{array}{l}\text { are collections of individuals } \\
\text { which define the size of the } \\
\text { population in the design of } \\
\text { genetic algorithm and create } \\
\text { the initial population } \\
\text { (randomly chosen) in the } \\
\text { beginning of genetic } \\
\text { algorithm. }\end{array}$ \\
\hline Recombination & $\begin{array}{c}\text { occurs between two } \\
\text { individuals at one single } \\
\text { point or multiple points at } \\
\text { the individuals. }\end{array}$ \\
\hline $\begin{array}{l}\text { Chromosome } \\
\text { Crossover }\end{array}$ & $\begin{array}{l}\text { Chromosome crossover } \\
\text { mechanism was followed to } \\
\text { recombine to chromosome. }\end{array}$ \\
\hline $\begin{array}{c}\text { Chromosome } \\
\text { Mutation }\end{array}$ & $\begin{array}{l}\text { acts by changing value(s) of } \\
\text { a single individual. Mutation } \\
\text { prevents the solution of } \\
\text { being trapped in local } \\
\text { optima. }\end{array}$ \\
\hline Reproduction & $\begin{array}{l}\text { produces clone of single or } \\
\text { group of good individuals. } \\
\text { Reproduction prevents from } \\
\text { losing good individual } \\
\text { through recombination. }\end{array}$ \\
\hline Generation & $\begin{array}{l}\text { is state of population, which } \\
\text { comes from removing the } \\
\text { weak individuals from } \\
\text { population and adding new } \\
\text { individual from population }\end{array}$ \\
\hline Replacement & $\begin{array}{l}\text { is the process in genetic } \\
\text { algorithm, which replaces } \\
\text { individual by other one in } \\
\text { the population. }\end{array}$ \\
\hline Termination criteria & $\begin{array}{l}\text { Criteria for stopping genetic } \\
\text { algorithm includes } \\
\text { Maximum generation, } \\
\text { Specific time and Unchanged } \\
\text { fitness value. }\end{array}$ \\
\hline
\end{tabular}

Table 1: Genetic Algorithm Operators

(Sources Sivanandam and Deepa (2008), Arifin S. (2011)

\section{Method}

This section explained the steps involved in carrying out this work. The steps include; data preparation, hybridization process; data classification and evaluation.

\subsection{Data Preparation}

This involves preparing the accumulated data for training. It can be regarded as the data mapping phase which has to do with matching the parameters of CPNN-GA to selected variables of cardholder's transaction data. The accumulated data was presented in the form acceptable by the model with respect to its parameter. This includes average daily spending, credit card (CC) Usage Frequency, Proxy Port check, IP Address Check, Authentication Type Check, CC Overdraft, $\mathrm{CC}_{\mathrm{type}}$ - (type of card); $\mathrm{CC}_{\mathrm{bb}}$ ( the balance available at bank of credit card);

CCfreq-number of times card used); $\mathrm{CC}_{\text {loc }}$ (location at which credit cards in the hands of fraudsters); $\mathrm{CCov}_{\text {the }}$ rate of overdraft time; $\mathrm{CCdsp}$.(the average daily spending amount); $\mathrm{CC}_{\mathrm{cb}^{-}}$(amount of cashback); $\mathrm{CC}_{\mathrm{cbfq}^{-}}$(frequency of cash back) 


\subsection{The CPNN-GA model}

GA optimization was interposed into CPNN framework in order to optimize the CPNN training parameters so that the best chromosome having optimal parameter settings can be obtained. An initial population size was assumed and fed into CPNN framework that performs the classification. Error function is the average error incurred when CPNN classified the input data. The objective was to minimize Error Function and obtain the best chromosome for an optimal set of CPNN parameters that was used for prediction purposes. The initial weights were randomly selected between 0 and 1 . Figure 3 showed the model of proposed CPNN-GA framework.

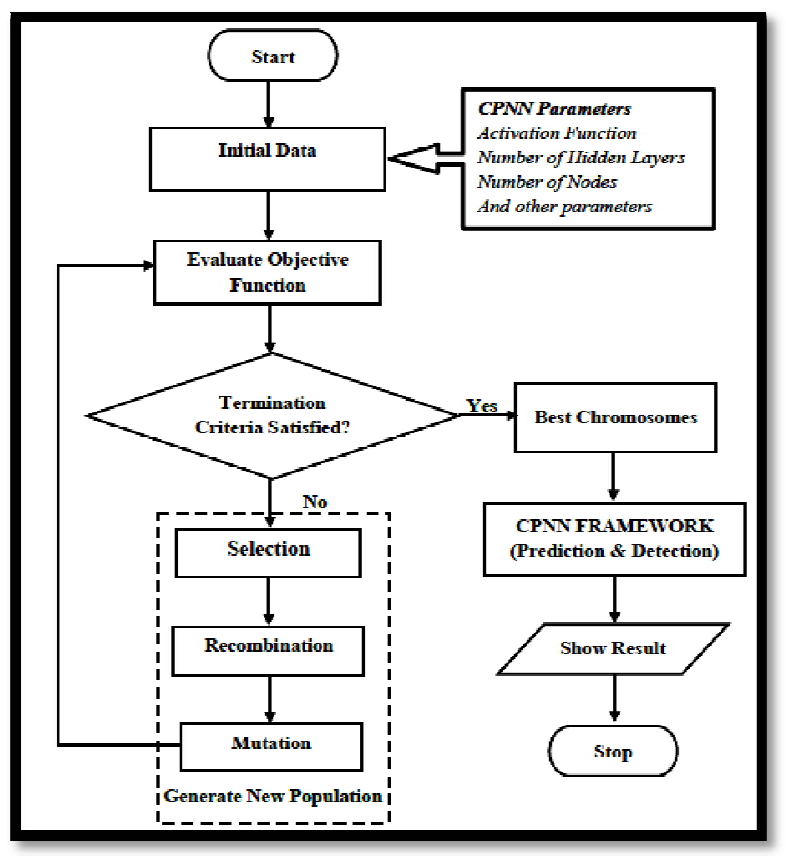

Figure 3: CPNN-GA model

\subsection{The Procedure of Genetic Algorithm}

This study developed genetic algorithm that tuned the CPNN with regards to determination of the network topology, determination of the set of input attributes and determination of the neuron weights. GA tried to optimize the network topology as it evaluates the genomes in its population for candidate network topologies, and tries to optimize that specific topology for set of input features. It also evaluated each of its own population members of candidate input feature combinations. For each of these input feature combinations, a CPNN test was constructed and trained. The construction took place for each candidate solution, given the fixed topology as determined by GA. In addition, GA optimized the weights for the constructed CPNN.

The entirety of parameters (input factors, topology, and weights) that were used was encoded into a single genome and tune by GA for optimization. For the encoding of the weights between neurons, this study chose a one-dimensional array of real numbers. The total length of the genome $L$ was calculated as

$$
L=n_{\text {input }} * n_{1}+\sum_{i=1}^{k-1}\left(n_{j} * n_{i+1}\right)+n_{k}
$$

Where " $n$ " input is the number of input attributes for the CPNN, $\mathrm{k}$ is the number of internal layers, and ni is the number of nodes in layer $\mathrm{i}$. The last term in the equation is for the weights between the last internal layer and the output layer, which consists of a single node. The encoding of the genome representing input features is done via simple binary encoding. A zero in a specific bit in the genome means an input attribute is not chosen for the CPNN design, whereas a one in that bit means that the input attribute is chosen for the CPNN design.

\subsection{Classification Process}

This phase entails the operations of classifying prepared data by GA into malicious and genuine transactions. The classification was performed by the hybridized CPNN-GA on the pre-processed dataset. The dataset was divided into; a training set and testing set. When the data sample was fraudulent, expected response of the CPNN-GA system was 1 , and produced 0 otherwise.

\subsection{Evaluation Metrics}

The metrics employed in this paper were shown in confusion matrix in table 2and the performance parameters are prediction accuracy $\left(\mathrm{p}_{\mathrm{acc}}\right)$, false acceptance rate $\left(\mathrm{fa}_{\mathrm{rate}}\right)$, false rejection rate $\left(\mathrm{fr}_{\text {rate }}\right)$, and equal error rate ( eqrate $_{\text {re }}$ ). 


\begin{tabular}{|c|c|c|}
\hline \multirow{2}{*}{ Actual } & \multicolumn{2}{|c|}{ Predicted } \\
\cline { 2 - 3 } & Positive class & Negative class \\
\hline Positive class & $\begin{array}{c}\text { True Positive } \\
\text { (IP) }\end{array}$ & $\begin{array}{c}\text { False } \\
\text { Negative (FN) }\end{array}$ \\
\hline Negative class & $\begin{array}{c}\text { False Positive } \\
\text { (FP) }\end{array}$ & $\begin{array}{c}\text { True Negative } \\
\text { (TN) }\end{array}$ \\
\hline
\end{tabular}

Table 2: Confusion Matrix

$\mathrm{fa}_{\mathrm{rate}}=$ false positive $/$ (false positive + true negative)

$\mathrm{fr}_{\text {rate }}=$ false negative $/$ (false negative + true negative)

Predictive Accuracy $=\frac{T P+T N}{T P+F P+T N+F N}$

Error Rate $=\frac{F P+F N}{T P+F P+T N+F N}$

\subsection{Implementation of the Detection System in MATLAB}

An interactive Graphic User Interface (GUI) were designed (as shown in figure 3) The implementation tool used was MATLAB R2012a version on Windows 7 Ultimate 32-bit operating system, intel CPU 2.20GHZ Central processing Unit, 4GB Random Access Memory and 500GB hard disk drive.

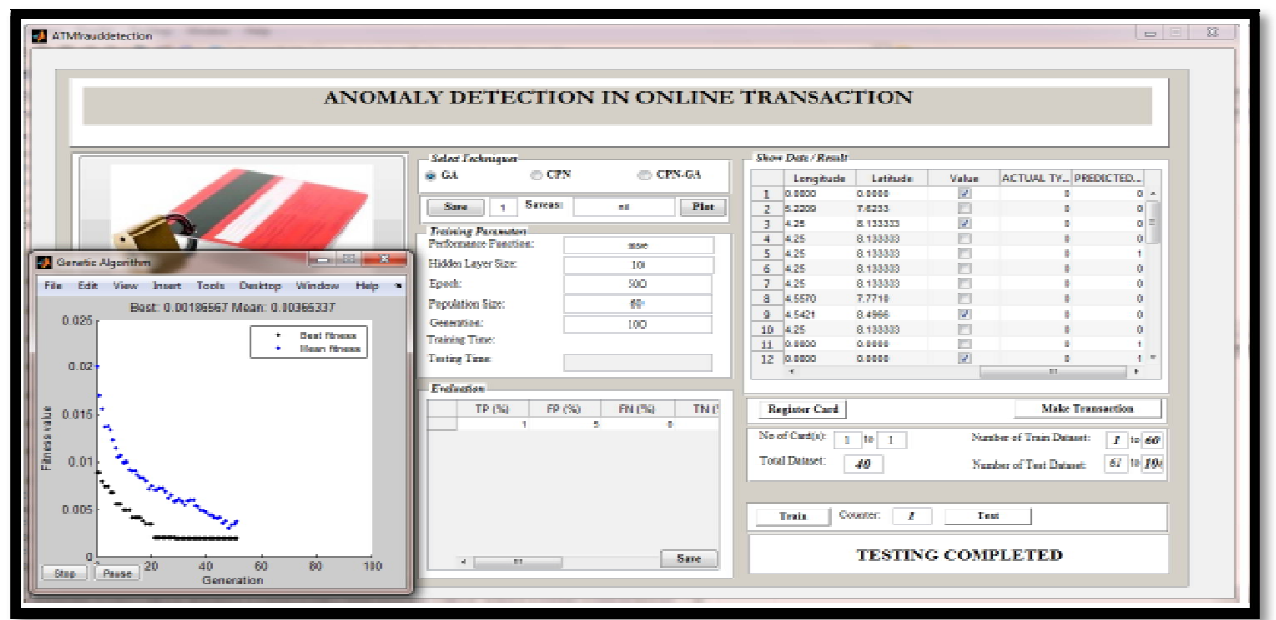

Figure 4: GUI for GA Anomaly Detection System

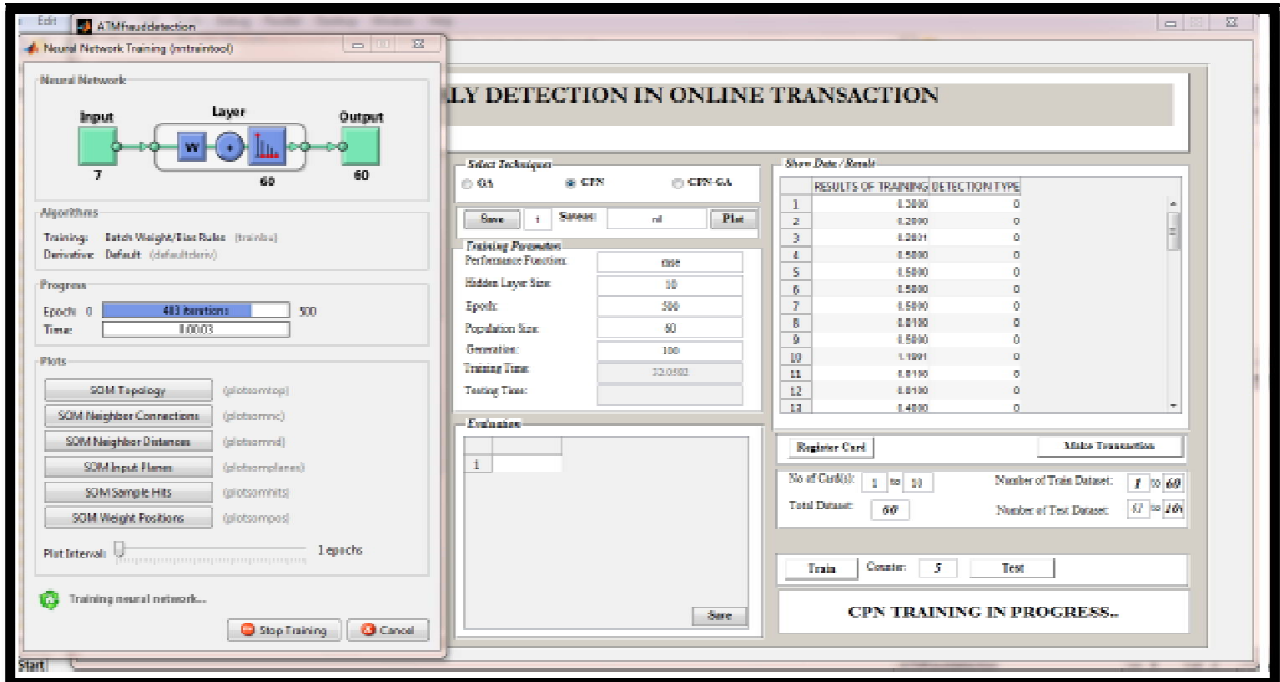

Figure 5: GUI for CPNN Anomaly Detection System 


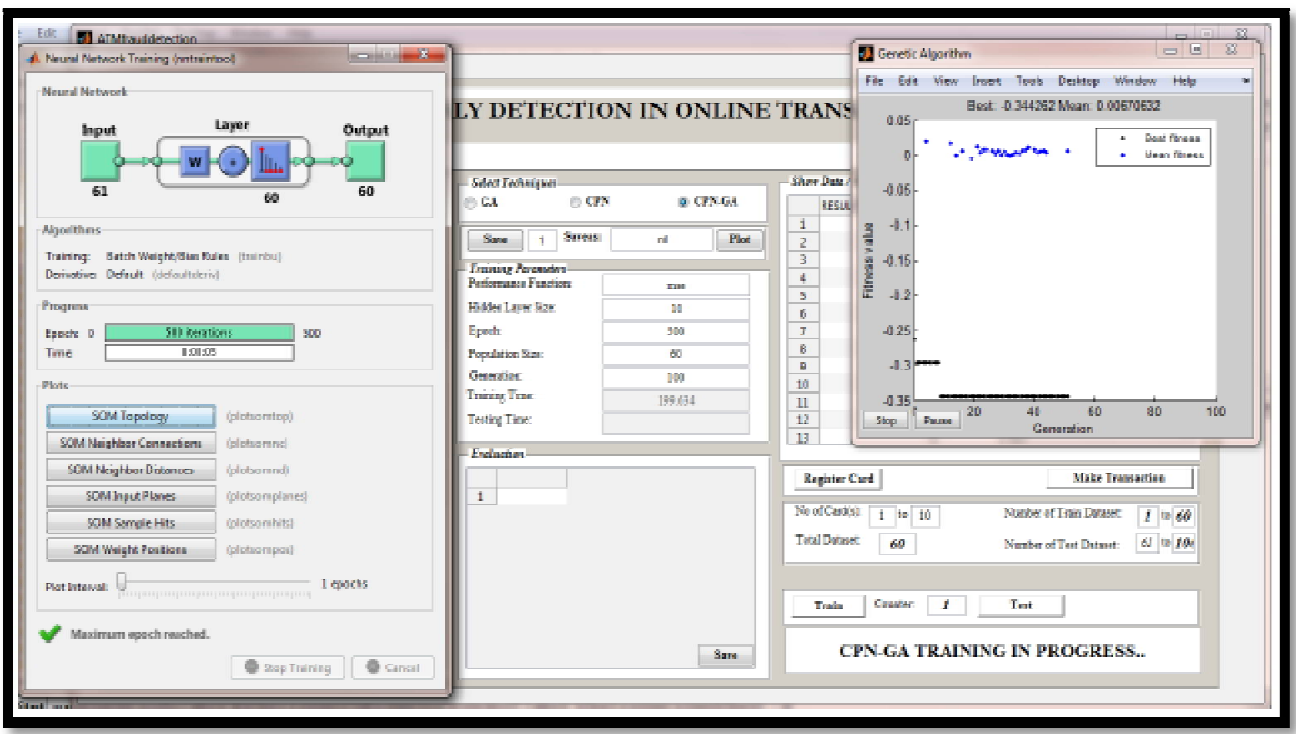

Figure 6: GUI for CPNN-GA Anomaly Detection System

\section{Results and Discussion}

A simulator was used to generate a total of 1,300 data intertwined of genuine and fraudulent transactions. Table 3 , 4 and 5 showed some of the results of GA, CPNN and CPNN-GA anomaly detection systems with metrics; prediction accuracy $\left(\mathrm{p}_{\text {acc }}\right)$, false acceptance rate $\left(\mathrm{fa}_{\text {rate }}\right)$, false rejection rate $\left(\mathrm{fr}_{\mathrm{rate}}\right)$, and equal error rate $\left(\mathrm{eq}_{\mathrm{rate}}\right)$. The averages of results generated by GA anomaly detection system were $95.0 \%, 0.0 \%, 10.0 \%$, and $5.0 \%$; CPNN anomaly detection system produced averages of $94.5 \%, 0.0 \%, 7.3 \%$ and $3.8 \%$ while CPNN-GA anomaly detection system gave $97.3 \%, 0.0 \%, 3.7 \%$ and $2.0 \%$ for $\mathrm{p}_{\text {acc, }}$ farate, $\mathrm{fr}_{\text {rate }}$ and eqrate respectively. The false alarm rate for the GA, CPNN and CPNN-GA are $0.83 \%, 1.35 \%$ and $0.61 \%$ respectively.

\begin{tabular}{|lllllllll|}
\hline Cards & $\begin{array}{l}\text { TP } \\
\text { (\%) }\end{array}$ & $\begin{array}{l}\text { FP } \\
(\%)\end{array}$ & $\begin{array}{l}\text { FN } \\
(\%)\end{array}$ & $\begin{array}{l}\text { TN } \\
(\%)\end{array}$ & $\begin{array}{l}\text { FRR } \\
(\%)\end{array}$ & $\begin{array}{l}\text { FAR } \\
(\%)\end{array}$ & $\begin{array}{l}\text { EER } \\
(\%)\end{array}$ & $\begin{array}{l}\text { ACC } \\
(\%)\end{array}$ \\
\hline 1 & 1 & 4 & 0 & 35 & 0 & 10.00 & 5.00 & 95.00 \\
2 & 2 & 3 & 0 & 35 & 0 & 7.50 & 3.75 & 96.25 \\
3 & 2 & 4 & 0 & 34 & 0 & 10.00 & 5.00 & 95.00 \\
4 & 0 & 5 & 0 & 35 & 0 & 12.50 & 6.25 & 93.75 \\
5 & 1 & 4 & 0 & 35 & 0 & 10.00 & 5.00 & 95.00 \\
\hline
\end{tabular}

Table 3: Analysis of Results Generated by GA

\begin{tabular}{|lllllllll|}
\hline Cards & $\begin{array}{l}\text { TP } \\
(\%)\end{array}$ & $\begin{array}{l}\text { FP } \\
(\%)\end{array}$ & $\begin{array}{l}\text { FN } \\
(\%)\end{array}$ & $\begin{array}{l}\text { TN } \\
(\%)\end{array}$ & $\begin{array}{l}\text { FRR } \\
(\%)\end{array}$ & $\begin{array}{l}\text { FAR } \\
(\%)\end{array}$ & $\begin{array}{l}\text { EER } \\
(\%)\end{array}$ & $\begin{array}{l}\text { ACC } \\
(\%)\end{array}$ \\
\hline 1 & 1 & 3 & 0 & 36 & 0 & 7.50 & 3.75 & 96.25 \\
2 & 1 & 2 & 0 & 37 & 0 & 5.00 & 2.50 & 97.50 \\
3 & 1 & 3 & 0 & 36 & 0 & 7.50 & 3.75 & 96.25 \\
4 & 0 & 4 & 0 & 36 & 0 & 10.00 & 5.00 & 95.00 \\
5 & 2 & 3 & 0 & 35 & 0 & 7.50 & 3.75 & 96.25 \\
\hline
\end{tabular}

Table 4: Analysis of Results Generated by CPNN

\begin{tabular}{|lllllllll|}
\hline Cards & $\begin{array}{l}\text { TP } \\
(\%)\end{array}$ & $\begin{array}{l}\text { FP } \\
(\%)\end{array}$ & $\begin{array}{l}\text { FN } \\
(\%)\end{array}$ & $\begin{array}{l}\text { TN } \\
(\%)\end{array}$ & $\begin{array}{l}\text { FRR } \\
(\%)\end{array}$ & $\begin{array}{l}\text { FAR } \\
(\%)\end{array}$ & $\begin{array}{l}\text { EER } \\
(\%)\end{array}$ & $\begin{array}{l}\text { ACC } \\
(\%)\end{array}$ \\
\hline 1 & 1 & 1 & 0 & 38 & 0 & 2.50 & 1.25 & 98.75 \\
2 & 3 & 2 & 0 & 35 & 0 & 5.00 & 2.50 & 97.50 \\
3 & 1 & 2 & 0 & 37 & 0 & 5.00 & 2.50 & 97.50 \\
4 & 0 & 1 & 0 & 39 & 0 & 2.50 & 1.25 & 98.75 \\
5 & 1 & 1 & 0 & 38 & 0 & 2.50 & 1.25 & 98.75 \\
\hline
\end{tabular}

Table 5: Analysis of Results Generated by CPNN-GA 


\section{Conclusion}

This paper presented an optimized CPNN with GA. CPNN is a hybridized scheme of neural network and self organizing map while GA optimized CPNN parameters. GAs are global search methods that are based on principles like selection, crossover and mutation. The averages of results generated by GA anomaly detection system were $95.0 \%, 0.0 \%$, $10.0 \%$, and $5.0 \%$; CPNN anomaly detection system produced averages of 94.5\%, $0.0 \%, 7.3 \%$ and 3.8\% while CPNN-GA anomaly detection system gave $97.3 \%, 0.0 \%, 3.7 \%$ and $2.0 \%$ for $\mathrm{p}_{\text {acc, }}, \mathrm{fa}_{\text {rate, }}, \mathrm{fr}_{\text {rate }}$ and eqrate respectively.A hybrid approach based on CPNN and GA provided results for enhanced accuracy in online transactions fraud detection system with lowest falsealarm rate.The developed fraud detection system is critically important for security and economic operation in banking system.

\section{References}

i. C. Varun, B. Arindam and K. Vipin (2009). Anomaly Detection: A Survey. ACM Computing Surveys, 41(3), pp 1-58.

ii. N.Noori, L.Boti and E.Shami (2012): "Surveying Different Aspects of Anomaly Detection and Its Applications". The Journal of Mathematics and Computer Science Vol. 4 No.2 Pp: 129 - 138.

iii. R.Patidar, and Sharma L. (2011) "Credit Card Fraud Detection Using Neural Network" In: International Journal of Soft Computing and Engineering (IJSCE), 1, 32-38.

iv. P. K.Chan, W.Fan, A. L.Prodromidis, and S. J. Stolfo, (1999). "Distributed data mining in credit card fraud detection." In: Intelligent Systems and their Applications, IEEE, 14(6), 67-74.

v. T. O. Oladele, O. A.Olajide and J. A. Emmanuel(2018). A Hybridized Semi-Global Sequence Alignment Algorithm for Intrusion-Detection Systems, Afr. J. Comp. \& ICT, Vol.11, No.3, pp. 44 - 70.

vi. A. Kundu, S. Panigrahi, S. Sural, and A. K. Majumdar, (2009). "BlAST--SSAHA hybridization for credit card fraud detection," IEEE Transactions on Dependable and Secure Computing, vol. 6, no. 4, pp. 309-315.

vii. A. John, (2013), "Data Mining Application for Cyber Credit-Card Fraud Detection System", Springer-Verlag Berlin Heidelberg, pp. 218-228.

viii. C. Pooja, A.D. Thakare, P. Kale, M. Gole, P. Nanekar (2015).Genetic K-means Algorithm for Credit Card Fraud Detection, (IJCSIT) International Journal of Computer Science and Information Technologies, Vol. 6 (2), 17241727.

ix. S.Panigrahi, A.Kundu, S.Sural, A. K.Majumdar, (2009): "Credit card fraud detection: A fusion approach using Dempster-Shafer theory and Bayesian learning" Special Issue on Information Fusion in Computer Security science direct Pp: 354-363.

x. T.Kohonen, (1995). Self-Organizing Map. 2nd edition, Berlin: Springer-Verlag. pp.1-12. 